\title{
Minimally Frozen Elephant Trunk
}

\author{
Adnan Ak, Davut Cekmecelioglu*, Ozgur Arslan, Mesut Sismanoglu and Mehmet Altug Tuncer \\ Department of Cardiovascular Surgery, Kartal Kosuyolu Yuksek Ihtisas Research and Training Hospital, Turkey
}

Received: May 28, 2018; Published: June 07, 2018

*Corresponding author: Davut Cekmecelioglu, Kartal Kosuyolu Yuksek Ihtisas, Egitimve Arastirma Hastanesi, Department of Cardiovascular Surgery Denizer Cevizli, Kartal, Istanbul, Turkey

\section{Abstract}

Background: While surgery involves high surgical mortality and morbidity in complex thoracic aortic diseases, surgical exposure is very important, and it is necessary to explore the area as wide as possible. Increased experience in minimal invasive surgery and advances in hybrid stent graft technologies allow us to perform easy and successful operations through a limited incision area. A 39year-old male patient applied to our clinic with hoarseness and back pain complaints, and was diagnosed with chronic Type-I aorta dissection.

Methods: We described how we treated the patient successfully by applying Bentall de Bono, arcus aorta restoration, and frozen elephant trunk procedures through mini j sternotomy. For better manipulation of the aortic arch branches, after the placement of 24/29Fr Carpentier two-stage bicaval femoral venous cannula from innominate vein through to inferior vena cava, left innominate vein was divided.

Results: Patient was discharged at 6th post-operative day without any neurological deficit and hoarseness. No upper extremity swelling or neurological complications were observed due to left innominate vein division.

Conclusion: With increasing usage of hybrid methods in aortic surgery, we believe that partial J mini-sternotomy implementations can be carried out successfully and safely in arc us aorta and proximal descending aorta operations, involving even type-1 aortic dissections.

Keywords: Minimally Invasive; FET; Innominate Vein Cannulation

\section{Introduction}

Complex thoracic aorta diseases can be treated successfully with acceptable mortality and morbidity by means of hybrid surgical techniques [1]. Especially because of its reliability in aortic root surgery, minimum post-operative complications and the short healing process, minimal invasive surgical methods [2], having higher popularity in recent two decades, would improve the success level of hybrid stent graft technology and provide us with better surgical results. In this paper, we described how we treated a patient diagnosed with Type-I aortic dissection successfully by applying Bentall de Bono, arcus aorta restoration, and frozen elephant trunk procedures through mini $\mathrm{j}$ sternotomy. We presented the surgical method, which we implemented, through a short literature review.

\section{Case}

A 38-year-old male patient with only a history of hypertension applied to our clinic with complaints of hoarseness and back pain. Patient's initial blood pressure was measured134/89 mmHg in the right arm and $140 / 94 \mathrm{mmHg}$ in the left arm. Peripheral pulses were palpable in four extremities. A sinus rhythm was observed in the ECG. Because of hoarseness, physical examinations were also performed in ear nose and throat clinic. No neurological or anatomical problem was found. Cranial computerized tomography (CT) of the patient was normal. In computerized tomographic angiography (CT-A), the sinus valsalva, ascending aorta and descending aorta were measured to be 40,41 and $66 \mathrm{~mm}$, respectively. Starting from the 2 nd to 3 rd distal part of ascending aorta and ending at iliac bifurcation, a dissection flap was observed, and all of the aortic branches, except the left renal artery, were originating from true lumen. A major re-entry was present in $3 \mathrm{~cm}$ distal to the left subclavian artery orifice. Aortic root was observed at the 3rd intercostal gap level. In transthoracic echocardiography, ejection fraction was $40 \%$. Aortic valve was in tricuspid configuration but due to an obvious coaptation failure, severe regurgitation was present. In coronary CT-A and myocardium perfusion scintigraphy, no coronary artery pathology was found. Surgical operation was planned under elective conditions. 


\section{Surgical Technique}

Complete monitorization was performed by placing central catheter from right jugular vein, arterial monitorization from left arm, cerebral pulse oximeter, and cerebrospinal fluid drainage catheter. After exploring the right subclavian artery, J-shaped mini-sternotomywas performed at the level of $4^{\text {th }}$ right intercostal gap. Following systemic heparinization, subclavian arterial cannulation was performed with CalMed 18Fr artery cannula. For better manipulation of the aortic arch branches, after the placement of $24 / 29 \mathrm{Fr}$ Carpentier two-stage bicaval femoral venous cannula from innominate vein through to inferior vena cava, left innominate vein was divided (Figure 1). CPB was initiated with a mean flow rate of 2.18 $\mathrm{L} / \mathrm{min} / \mathrm{m} 2$ during the operation. A venting cannula was inserted through right superior pulmonary vein. Myocardial protection was ensured with antegrade blood cardioplegia.

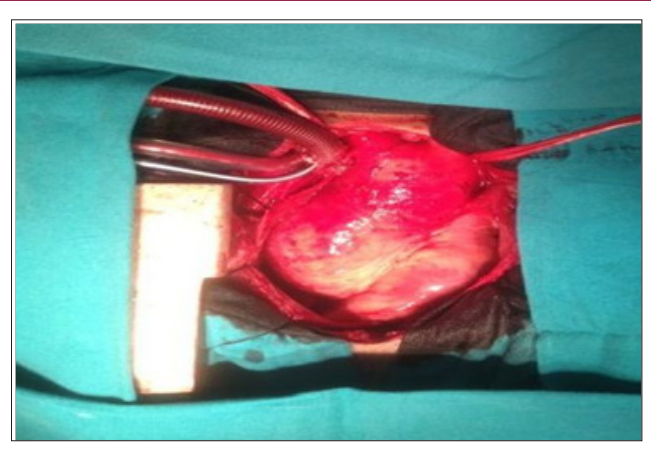

Figure 1: Innominate Vein Cannulation with Mini J partial sternotomy.

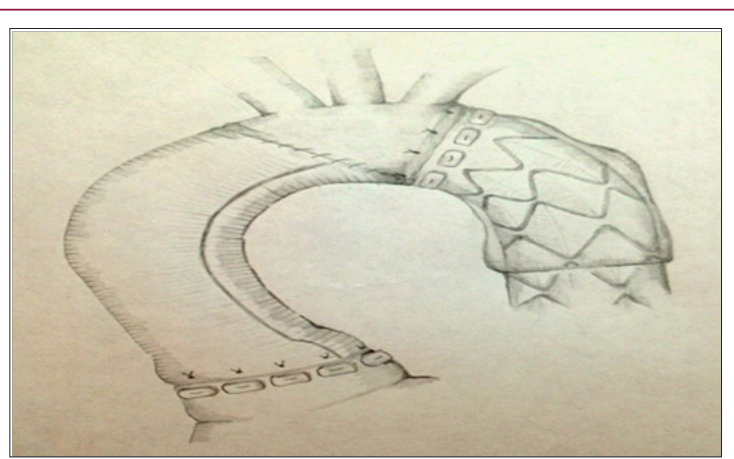

Figure 2: Illustration of Frozen Elephunt Trunk technique.

Following aortotomy, the main tear was observed right at the middle segment of ascending aorta. The aortic valve had 3 leaf lets, nevertheless the valve was resected due to the presence of coaptation defect secondary to annular enlargement. Afterwards Bentall de Bono procedure was implemented with Mitro flow valsalva conduit graft (Sorin group) (23 no valve and 28 no graft). Trans-esophageal temperature was decreased to $280 \mathrm{C}$ and after changing the position of X clamp from aorta to innominate artery, anterograde selective cerebral perfusion was initiated with a flow rate of $10-15 \mathrm{~mL} / \mathrm{kg} / \mathrm{min}$. Following the insertion of the $25 \mathrm{mmx}, 25 \mathrm{mmx}$ $150 \mathrm{~mm}$ E-Vita Open Plus graft (JOTEC $® G m b H$, Germany) through true lumen with the guidance of trances ophageal ehocardiography, proximal edge of the stent graft was fixed circumferentially at the level of $1 \mathrm{~cm}$ distally to the left subclavian artery orifice. The
E-vita was cut so the inner graft remained $2 \mathrm{~cm}$. After the graft was pre-anastomosed to the proximal aorta, the islet-shaped arcs elements and E-vita Open plus prosthesis were anastomosed to each other (Figure 2). The patient was discharged at the 6th post-operative day without any neurological deficits or clinical and radiological pathologies (Figure 3).

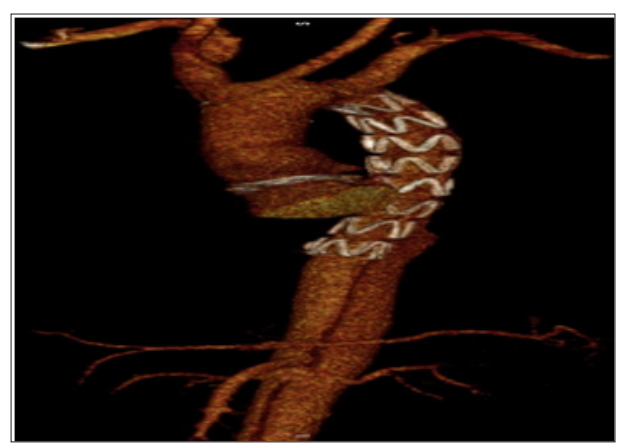

Figure 3: The final image of the operation.

\section{Discussion}

In complex aortic diseases, frozen elephant trunk procedure can be implemented more easily with acceptable mortality [1-2]. In the last two decades, with increasing experience in minimal invasive surgery and hybrid methods, easier therapy modalities improving the comfort of the patients has been increasingly utilized. In this paper, we reported single-phased minimally invasive restoration of aortic root and thoracic aorta, arcus replacement and frozen elephant trunk procedure on a patient, who has been diagnosed with chronic Type-I aortic dissection. The entire procedure, in order to obtain better results in short- and long-term, was performed using J-shaped partial sternotomy. In the treatment of aortic root and ascending aorta diseases, it has been corroborated by many studies that surgical procedures involving mini-sternotomy provide sufficient view and safety and patients recover in shorter time with less drainage and blood transfusion requirement [3-4].

In order to obtain larger and effective surgical area and to ensure surgical comfort for aortic root procedures, dual-stage venous cannula was placed through the proximal part of the left innominate vein towards the right atrium, and then the innominate vein was divided [5]. Without any need for an additional cannula from right atrium appendix or femoral vein necessitating an additional incision, innominate vein cannulation provided sufficient flow. In case of any in nominate vein injuries that may occur due to the tractions and dissections during the operation or when it is required to divide the left in nominate vein in arcus aorta interventions for a better surgical exposure, it has been reported that there is a risk of swelling of the upper-left extremity or cerebral edema leading to neurological manifestations, both due to venous return insufficiency, yet those adversities can be resolved with elevation [6]. In our case, no upper extremity swelling or neurological complications were observed after frozen elephant trunk procedure and left in nominate vein division. Unfortunately, we don't have an extensive experience on this minimally invasive procedure, which we present through a single patient only. But with increasing usage of hybrid methods in aortic surgery, we believe that partial J mini-sternotomy 
implementations can be carried out successfully and safely in arcus aorta and proximal descending aorta operations, involving even type- 1 aortic dissections.

\section{References}

1. Tian DH, Wan B, Di Eusanio M, Yan TD (2013)Systematic review protocol: the frozen elephant trunk approach in aortic arch surgery.Ann Cardiothorac Surg 2(4):578.

2. Akbulut M, Arslan O, Ak A, Tas S, Cekmecelioglu D, Donmez AA, et al. (2017) Early period results for repair of complex thoracic aortic diseases with E-vita open stent graft. EJCM 05 (1): 1-6.

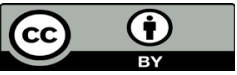

This work is licensed under Creative Commons Attribution 4.0 License

Submission Link: https://biomedres.us/submit-manuscript.php
3. Sun L, Zheng J, Chang Q, Tang Y, Feng J, et al. (2000) Aortic Root Replacement by Ministernotomy-Technique and Potential Benefit.Ann ThoracSurg 70(6):1958-1961.

4. Perrotta S, Lentini S (2009) Ministernotomy approach for surgery of the aortic root and ascending aorta. Interact Cardiovasc. Thorac Surg 9(5): 849-858.

5. Chan EY, Lumbao DM, Iribarne A, Easterwood R, Yang JY, Cheema FH, et al. (2012) Evolution of Cannulation Techniques for Minimally Invasive Cardiac Surgery A 10-Year Journey.Innovations (Phila) 7(1):9-14.

6. McPhee A, Shaikhrezai K, Berg G(2013) Is it safe to divide and ligate the left innominate vein in complex cardiothoracic surgeries?.Interact Cardiovascular Thorac Surg 17(3):560-563.

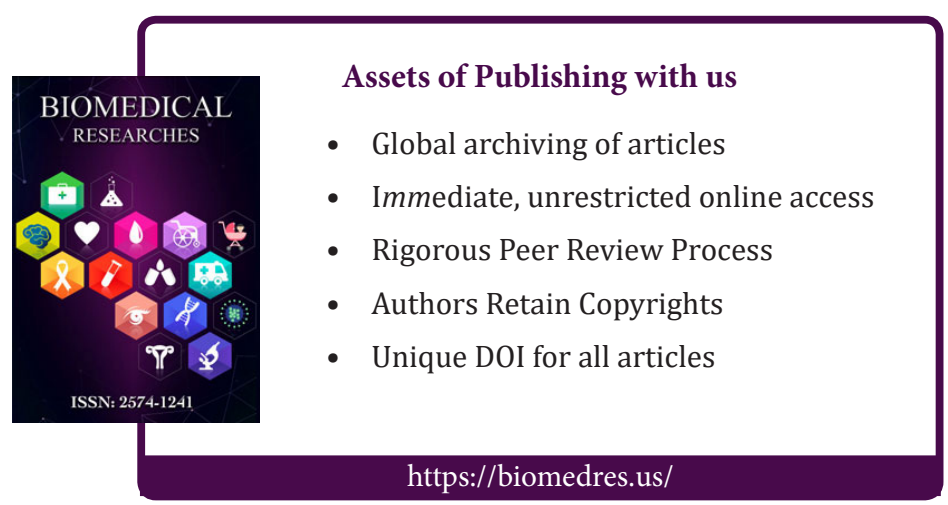

